\title{
COMPUTING LEAD-FIELD MATRICES FOR A BODY TISSUE CONDUCTIVITY RANGE
}

\author{
Jones $\mathrm{RT}^{1}$, Schulze WHW ${ }^{1}$, Potyagaylo $\mathrm{D}^{1}$, Dössel $\mathrm{O}^{1}$ and Weber $\mathrm{FM}^{1,2}$ \\ ${ }^{1}$ Institute of Biomedical Engineering, Karlsruhe Institute of Technology, Karlsruhe, Germany \\ ${ }^{2}$ Philips Research Europe, Hamburg, Germany \\ publications@ibt.kit.edu
}

\begin{abstract}
A new method to predict changes in a lead-field matrix induced by conductivity variations of a single body tissue is proposed. The approach is based on the principle component analysis (PCA) with three initial lead-field matrices transformed to vectors as input. For each tissue - blood, lungs, muscles and fat - a PCA was carried out. Further, for each tissue the default conductivity value and the conductivity varied by $\pm 50 \%$ were used to calculate the sample lead-field matrices. The results of the PCAs indicate that for every tissue the first principle component suffices to predict the conductivity-induced changes in the samples. With an interpolation of the scores we additionally show that the prediction is not bound to the sample matrices but moreover every matrix within each conductivity range is possibly estimated and conclusively predicted.
\end{abstract}

Keywords: inverse problem of electrocardiography, leadfield matrix, tissue conductivities, PCA

\section{Introduction}

Overcoming the inverse problem of ECG imaging promises better non-invasive cardiac diagnosis and real-time monitoring of the electrical activities of the heart. An early step of the problem is to accurately determine the lead-field matrix, which is part of the forward problem in ECG imaging. It linearly connects the cardiac electrical activity to the body surface potentials. The so-called forward calculation of a lead-field matrix is time consuming with high computational costs. Further, its initial requirements are a human body segmentation and accurate body tissue conductivities. However, the literature values of these conductivities are not consistent [1]. Changes in lead-field matrices due to conductivity changes must be taken into account by repeating the time-consuming forward calculations.

In this work we used the principal component analysis (PCA) to bypass these inefficiencies. The method discussed in this paper is adopted from Weber et al. [2] in which the influences of the conductivities have been studied in the context of ECG forward calculations. The PCA method reduces the dimensionality of a dataset without significant loss of information.

As initial input for the PCA we generated three sample matrices with a wide range of conductivities for one single tissue. The result of the PCA allows us to simplify the reconstruction by only using the first principal component. With an interpolation of the conductivity-dependent PCA scores all lead-field matrices within the chosen conductivity range are easily reconstructed.

\section{Methods}

The computer simulations in this study use the Visible Man dataset as model. In these simulations 64 electrodes are used for measuring the body surface potential and a tetrahedron model with 2143 nodes is fitted into the ventricular myocardium. Thus, each lead-field matrix is made up of 2143 columns and 64 rows. We varied the conductivity of blood $B$, lungs $L$, muscles $M$ and fat $F$ which are all referred to as tissues. As initial conductivities for these tissues the values from Gabriel et al. [3] (GG) were used. Further, $+50 \%$ and $-50 \%$ of the respective GG value are used for each tissue.

The so-called forward calculation calculates the body surface potentials from the electrical sources in the heart created using the bidomain model [4]. In order to determine a lead-field matrix, one single forward calculation is executed for every single myocardial node. In other words, every node is subsequently turned on with all the others turned off. The results of every node are overlaid and thus a leadfield matrix is obtained. For a new set of conductivities the whole procedure has to be repeated.

For each of the four tissues a PCA was applied separately. We passed three lead-field matrices to every PCA as input data. One of them was reused in every PCA. Thus, a total of nine lead-field matrices with different conductivity compositions were calculated, so that every tissue has three corresponding matrices with its conductivity set to $\pm 50 \%$ and $G G$. In order to perform a PCA those three matrices are transformed into long row vectors by putting every row in-line. The PCA input matrix is made up of the transpose of these three vectors as columns.

As the input data of the PCA only yields a minor degree of freedom it is executed with a method based on the singular value decomposition. This more efficient method results in the first $n-1$ principal components $\vec{p}_{j}$ where $n$ is the number of input columns of the PCA. Additionally, the PCA returns a mean vector $\bar{x}$ of the input matrix, variance values $\lambda_{j}$ for every principal component and a score matrix $S$ with score values $s_{j, i}$ for every principle component $j$ and input point $i$. For the three input points $\left(\sigma_{i}= \pm 50 \%\right.$, $G G ; n=3$ ) this method returns two principle components. The reconstruction of the sample vectors $\vec{x}_{\sigma_{i}}$ is as follows:

$$
\vec{x}_{\sigma_{i}} \approx \bar{x}+s_{1, i} \cdot \vec{p}_{1}+s_{2, i} \cdot \vec{p}_{2}
$$




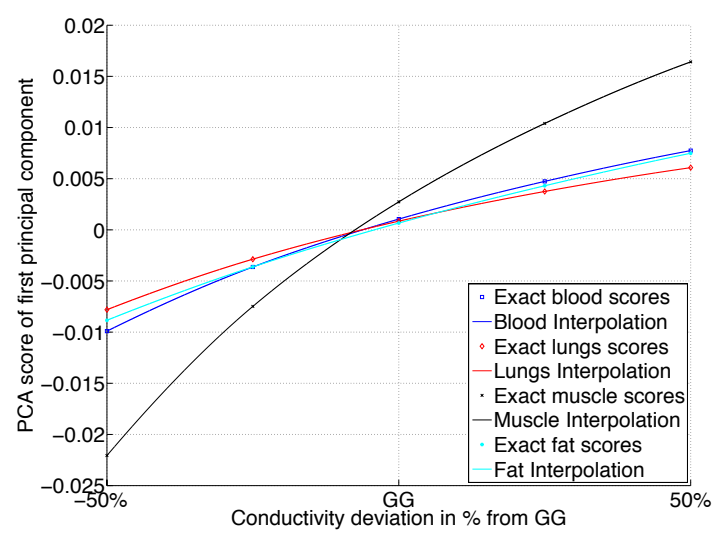

Figure 1: Scores of the first principle component and their interpolation.

Due to $\lambda_{1} \gg \lambda_{2}$ as shown in the results only the first principal component $\vec{p}_{1}$ is considered from now on. Moreover the true score values are replaced by a function of their interpolation $s\left(\sigma_{i}\right)$. Now the reconstruction not only allows to calculate the sample vectors but any vector with the tissue conductivity set in between $\pm 50 \%$.

$$
\vec{x}_{\sigma_{i}} \approx \bar{x}+s\left(\sigma_{i}\right) \cdot \vec{p}_{1}
$$

Finally, the reconstructed vectors $\vec{x}$ are compared to their real counterpart $\vec{x}_{\text {ref }}$ with the relative error formula 3 . As the sample data, every $\vec{x}_{\text {ref }}$ is calculated in a long-term forward calculation.

$$
\text { error }_{\text {matrix }}=\frac{\left\|\vec{x}-\vec{x}_{\text {ref }}\right\|_{2}}{\left\|\vec{x}_{\text {ref }}\right\|_{2}}
$$

Furthermore, the angles of the first principle components of the different PCAs are compared to estimate whether an approach combining the conductivity interactions is reasonable.

\section{Results}

The rate between the first and second principle component variances $\frac{\lambda_{1}}{\lambda_{2}}$ of the respective PCA is $167,760,592$ and 754 for blood, the lungs, muscle and fat.

Table 1 shows the relative errors of reconstructions within a single tissue. These reconstructions use equation 2 with the previously forward-calculated true scores or interpolated scores according to the conductivity value.

The angles between the first principle components of all tissues range from $57^{\circ}$ to $114^{\circ}$.

\section{Discussion}

This paper proposes a method to determine lead-field matrices without the prior knowledge of the body tissue conductivities. It leads to a closed form formula with solely these conductivities as variables. A corresponding lead-field matrix can be obtained immediately with knowledge of real conductivities.
Table 1: Relative errors of the PCA generated matrices with single conductivity variation. The reconstructions used interpolated $(i)$ scores or true $(t)$ scores.

\begin{tabular}{llllll}
\hline & $\mathbf{- 5 0} \%$ & $\mathbf{- 2 5} \%$ & GG & $\mathbf{+ 2 5 \%}$ & $\mathbf{+ 5 0 \%}$ \\
\hline Blood & $0.36 \%_{t}$ & $0.98 \%_{i}$ & $0.90 \%_{t}$ & $0.33 \%_{i}$ & $0.54 \%_{t}$ \\
Lungs & $0.13 \%_{t}$ & $0.35 \%_{i}$ & $0.33 \%_{t}$ & $0.16 \%_{i}$ & $0.21 \%_{t}$ \\
Muscle & $0.30 \%_{t}$ & $1.19 \%_{i}$ & $1.04 \%_{t}$ & $0.57 \%_{i}$ & $0.78 \%_{t}$ \\
Fat & $0.15 \%_{t}$ & $0.37 \%_{i}$ & $0.39 \%_{t}$ & $0.19 \%_{i}$ & $0.24 \%_{t}$ \\
\hline
\end{tabular}

Table 1 shows the reconstruction errors of every tissue with the true scores in the columns $-50 \%$, GG and $+50 \%$. This small error is introduced by the negligence of all principle components except the first. The errors of the lungs reconstruction are the lowest which is reasonable because its rate between the first and second principle component is the highest. The errors in column $-25 \%$ and $+25 \%$ of table 1 cover an additional error which is caused by the interpolation of the scores shown in figure 1. The heretofore maximum error is $1.19 \%$. Thus, for one single tissue the assumption is valid that any lead-field matrix within the sample range is reconstructed sufficiently with the first principle component and interpolated score values.

The angles between the particular first principle components are all larger than $56.91^{\circ}$. Although these principle components are not strictly orthogonal, an approach of combining the conductivity influences ought to be possible. However, more profound investigations would exceed the scope of this paper.

In conclusion, this method calculates lead-field matrices more efficiently and is a promising approach to overcome the large computational costs for conductivity adjustments in clinical practice.

\section{Acknowledgement}

This project was funded by the German Research Foundation under grants DO637/10-1 and DO637/13-1.

\section{Bibliography}

[1] D. U. J. Keller et al., "Ranking the influence of tissue conductivities on forward-calculated ecgs," IEEE Trans. Biomed. Eng., vol. 57, pp. 1568-1576, 2010.

[2] F. M. Weber et al., "Predicting tissue conductivity influences on body surface potentials-an efficient approach based on principal component analysis," IEEE Trans. Biomed. Eng., vol. 58, pp. 265-273, 2011.

[3] S. Gabriel et al., "The dielectric properties of biological tissues: Ii. measurements in the frequency range $10 \mathrm{hz}$ to 20 ghz," Physics in Medicine and Biology, vol. 41, pp. 2251-2269, 1996.

[4] D. B. Geselowitz and T. W. Miller, "A bidomain model for anisotropic cardiac muscle," Annals of Biomedical Engineering, vol. 11, pp. 191-206, 1983. 